\title{
Riesgo de mantener obesidad desde la infancia hasta el final de la adolescencia
}

\author{
MR. Albañil Ballesteros ${ }^{a}$, ME. Rogero Blanco ${ }^{b}$, M. Sánchez Martínc, \\ A. Olivas Domínguez $z^{\mathrm{a}}$, A. Rabanal Basalob, MT. Sanz Bayona ${ }^{\mathrm{c}}$ \\ aPediatra. CS Cuzco. Fuenlabrada, Madrid. España. \\ ${ }^{\mathrm{b}}$ Médico de Familia. CS Cuzco. Fuenlabrada, Madrid. España. \\ 'Enfermera. CS Cuzco. Fuenlabrada, Madrid. España.
}

\section{Resumen}

Introducción: la obesidad es uno de los principales problemas de salud dada su asociación, a cualquier edad, con una extensa comorbilidad, destacando la enfermedad cardiovascular y la diabetes tipo 2. Su presencia en la infancia supone un riesgo de persistencia en edades posteriores. El objetivo de este estudio es conocer el riesgo que tienen los niños que son obesos según el Índice de Masa Corporal (IMC), a distintas edades, de mantener este estado cuando son jóvenes adultos.

Pacientes y métodos: se realiza un estudio longitudinal de 153 niños nacidos en 1989, determinando su percentil de IMC por edad y sexo, a los 2, 3, 4, 6, 8, 11 y 14 años, e IMC a los 18 años. Se calcula el riesgo relativo que presentan los niños que son obesos a cada una de estas edades de presentar obesidad a los 18-19 años.

Resultados: los niños que presentan obesidad a partir de los seis años tienen un riesgo claro (de 7 a 23) de mantener obesidad a los 18 años, siendo máximo este riesgo $(23,8)$ a los 11 años: 40,9 en niñas y 11,7 en niños.

La prevalencia de obesidad a los 18 años es del 7,18\%, (intervalo de confianza del 95\% [IC 95\%]: 3,0-11,0) $(6,25 \%$ en hombres y $7,86 \%$ en mujeres) y la de sobrepeso es del 18,3\%, (IC 95\%: 11,9-24,0), (26,56\% en hombres y $12,35 \%$ en mujeres).

Conclusiones: los niños que son obesos a partir de los seis años presentan riesgo de ser obesos a los 18-19 años.

Palabras clave: Obesidad infantil. Sobrepeso infantil. Prevalencia. Índice de masa corporal. Seguimiento. Niños. Adolescentes. Atención Primaria.

The risk of staying obese from childhood to the end of adolescence

Abstract

Introduction: obesity is one of the most important problems in public health. It's associated, at any age, with extensive comorbidity, notably cardiovascular disease and type 2 diabetes.

When present in childhood, obesity poses a risk of remaining at later ages.

María Rosa Albañil Ballesteros, mralba100@hotmail.com

Los autores declaran no presentar conflictos de intereses en relación con la preparación y publicación de este artículo. 
The objective of this study is to know the risk of children who are obese (according to Body Mass Index: BMI) at different stages of childhood, to stay obese when they become young adults.

Subjects and methods: a long-term study of 153 children born in 1989 is carried out, calculating their $B M I$ at 2, 3, 4, 6, 8, 11, 14, and 18 years old. The relative risk of the children who are obese at each one of those ages to remain obese at 18-19 was calculated.

Results: children who are obese at 6, or later, showed an evident risk (from 7 to 23) to stay obese at 18. This risk peaks (23.8) at 11 years old, with figures of 40.9 in girls and 11.7 in boys.

The prevalence of obesity at 18 is $7.18 \%$ (Cl: $3.0-11.0)(6.25 \%$ in males, and 7.86 in females); the prevalence of overweight is $18.3 \%$ (CI: 11.9-24.0) (26.56\% in males and $12.35 \%$ in females).

Conclusions: children who are obese at age 6, or older, show a higher risk of remaining obese at 18-19 years old.

Key words: Childhood obesity. Childhood overweight. Prevalence. Body mass index. Tracking. Children. Adolescents. Primary care.

\section{Introducción}

Según la Organización Mundial de la Salud (OMS), la obesidad es actualmente uno de los principales problemas de salud pública. Su presencia se asocia a numerosas patologías, entre las que cabe destacar la enfermedad cardiovascular y la diabetes tipo $2^{1}$, no solo en la edad adulta ${ }^{2,3}$, sino también en la infan$\mathrm{cia}^{4-7}$. Afecta a ambos sexos, a todas las edades $y$, aunque algunos colectivos étnicos y poblacionales presentan mayor riesgo ${ }^{8}$, alcanza una distribución mundial, en sociedades desarrolladas y en desarrollo $0^{9,10}$ y aparece ligada a determinados estilos de vida ${ }^{11}$. El aumento de su prevalencia es exponencial en los últimos años y la estimación para el futuro tiende también al aumento, salvo pa- ra África ${ }^{12}$, a pesar de lo cual permanece infradiagnosticada ${ }^{13}$.

España no es una excepción, y a cualquier edad, niños, adultos y ancianos, registra una prevalencia de las más altas de Europa ${ }^{14}$. Como criterio diagnóstico de obesidad, se acepta internacionalmente, desde los dos años de edad, la determinación del índice de masa corporal (IMC), que se obtiene al dividir el peso en kilogramos entre la talla al cuadrado en metros $\left(\mathrm{kg} / \mathrm{m}^{2}\right)^{15}$. En adultos a partir de los 18 años, se considera sobrepeso la presencia de un IMC mayor o igual a 25 y menor de 30, y obesidad un IMC mayor o igual a 30. En niños se usan tablas de IMC, utilizando como punto de corte el valor correspondiente a los percentiles 95, 97 o 98 para definir 
obesidad y el valor correspondiente a los percentiles 85, 90 o 91 para definir sobrepeso, para cada edad y sexo ${ }^{16}$.

El sobrepeso y la obesidad en la infancia tienen un alto riesgo de perpetuarse en la adolescencia y la edad adulta ${ }^{2,17-19}$, y se relaciona una mayor intensidad del sobrepeso con un inicio más precoz ${ }^{20}$.

En publicaciones anteriores hemos recogido la prevalencia de obesidad y sobrepeso ${ }^{21}$ y la evolución del IMC ${ }^{22}$ de una muestra de población seguida desde los dos a los 14 años de edad. Actualmente, nuestro objetivo es conocer la frecuencia de obesidad y sobrepeso según el IMC a la edad de 18 a 19 años de una cohorte de jóvenes nacidos en 1989 y seguidos en un centro de salud, y conocer el riesgo de presentar obesidad a esta edad, si eran obesos a edades previas y para cada una de las edades consideradas.

\section{Pacientes y métodos}

Se trata de un estudio longitudinal prospectivo de cohorte. $Y$ longitudinal retrospectivo para riesgos. La población de estudio está formada por los nacidos en 1989, seguidos desde los dos años en las consultas de Pediatría del centro de salud Cuzco de Fuenlabrada, Madrid. Es una muestra total de 277 personas. Pertenecen a una población total de 26000 personas atendidas en el centro de salud Cuzco, según datos de tarjeta sanitaria a 31 de diciembre de 2007.

Fuenlabrada es una población del sur de la Comunidad de Madrid. Según datos del censo, en el año 2008 tenía 194791 habitantes y 122,60 de cada 1000 empadronados eran extranjeros. La renta per capita en 2007 fue de 12 629,03 euros (inferior a la media de la Comunidad de Madrid).

Se consideró criterio de exclusión la existencia de embarazo e hipotiroidismo no controlado y se valoró la existencia de trastornos del comportamiento alimentario.

Las variables consideradas fueron: edad (años cumplidos); sexo; origen étnico; existencia de intervención sobre el control del sobrepeso y obesidad, que se clasificó en tres modalidades (educativa, si se dio consejo; seguimiento, si se estableció un plan de actuación; y control y farmacológica, si se administraron fármacos); peso y talla a los 18-19 años, medidos con básculas SECA con tallímetro incorporado, con precisión de +/$100 \mathrm{~g}$ y hasta el milímetro más próximo, con el paciente descalzo, vestido con ropa interior, en bipedestación, haciendo coincidir su línea media sagital con la línea media del tallímetro y con la cabe- 
za en plano horizontal nariz-trago, tomados a los 2, 3, 4, 6, 8, 11 y 14 años; e IMC. Se consideró obesidad un IMC igual o mayor a 30 y sobrepeso un IMC mayor o igual a 25 y menor de 30 a partir de los 18 años de edad. Para las edades inferiores se consideró obesidad el valor correspondiente al percentil 95 y superiores de las tablas del Centers for Disease Control and Prevention (CDC) 2000, y sobrepeso los valores iguales o superiores al percentil $85 \mathrm{e}$ inferiores al percentil 95.

Los parámetros antropométricos se recogieron en el centro de salud por los miembros del equipo investigador.

Tras contactar por teléfono con los sujetos de la cohorte, se les proporcionó información por escrito y firmaron un consentimiento informado para su inclusión en el estudio. El trabajo se desarrolló a lo largo de 2008, con la aprobación y autorización de la Comisión de Investigación y del Comité Ético del Área 9 del Servicio Madrileño de Salud.

Para el análisis estadístico se utilizó el Programa SPSS ${ }^{\circledR}$ 15.0. Se calculó la prevalencia de obesidad y sobrepeso a los 18 años con el intervalo de confianza del 95\% (IC 95\%). Para calcular la magnitud de la asociación entre las variables en el estudio prospectivo de cohorte se utilizó el riesgo relativo (RR).

\section{Resultados}

De los 277 casos iniciales, en 50 no pudo establecerse contacto, 69 rechazaron participar y cinco fueron excluidos: tres por embarazo, uno por trastorno del comportamiento alimentario y uno por hipotiroidismo diagnosticado en el momento del estudio. La muestra final la componen 153 personas.

De ellas 64 , son $(41,8 \%)$ hombres y 89 $(58,2 \%)$ mujeres, el $96,7 \%$ de origen español, el $2 \%$ de origen sudamericano y el $1,3 \%$ de origen subsahariano. Todos habían cumplido 18 años y algunos 19 .

A los 18-19 años la prevalencia de obesidad es del 7,18\%, (IC 95\%: 3,0$11,0)(6,25 \%$ en hombres y $7,86 \%$ en mujeres) y la de sobrepeso es del 18,3\%, (IC 95\%: 11,9-24,0), (26,56\% en hombres y $12,35 \%$ en mujeres). En total, un $25,49 \%$ de la muestra presentó un $I M C \geq 25$. En 31 personas $(20,26 \%)$ se había producido una intervención educativa previa para corregir un estado de sobrepeso u obesidad. En la tabla 1 figuran las cifras de prevalencia de obesidad y sobrepeso e intervención educativa previa por sexos y tipo de intervención.

Las cifras de obesidad y sobrepeso en los sujetos de la muestra a todas las edades consideradas son como se detalla en la tabla 2, con una mayor preva- 
Tabla 1. Cifras de prevalencia de obesidad y sobrepeso, según IMC e intervención educativa previa por sexos y tipo de intervención

\begin{tabular}{l|c|c|c}
\hline & $\begin{array}{c}\text { Total } \\
\mathrm{N}=153(\%)\end{array}$ & $\begin{array}{c}\text { Hombres } \\
\mathbf{N}=64(\%)\end{array}$ & $\begin{array}{c}\text { Mujeres } \\
\mathbf{N}=89(\%)\end{array}$ \\
\hline Obesidad y sobrepeso según IMC & $28(18,3)$ & $17(26,6)$ & $11(12,4)$ \\
\hline Sobrepeso (IMC) & $11(7,2)$ & $4(6,3)$ & $7(7,9)$ \\
\hline Obesidad (IMC) & $114(74,5)$ & $43(67,2)$ & $71(79,8)$ \\
\hline Normopeso (IMC) & $39(25,5)$ & $21(32,8)$ & $18(20,2)$ \\
\hline Sobrepeso + obesidad (IMC) & & & \\
\hline Intervención educativa previa & $31(20,3)$ & $9(14,1)$ & $22(24,7)$ \\
\hline Intervención educativa previa & $15(9,8)$ & $5(7,8)$ & $10(11,3)$ \\
\hline Consejo & $15(9,8)$ & $3(4,7)$ & $12(13,5)$ \\
\hline Seguimiento & $1(0,7)$ & $1(1,6)$ & 0 \\
\hline Fármacos & $122(79,7)$ & $55(85,9)$ & $67(75,3)$ \\
\hline No intervención & & &
\end{tabular}

IMC: índice de masa corporal; $\mathrm{N}$ : tamaño muestral.

Tabla 2. Cifras de obesidad y sobrepeso en los sujetos de la muestra a todas las edades consideradas

\begin{tabular}{l|c|c|c|c|c|c|c}
\hline & & \multicolumn{3}{|c|}{ Sobrepeso (\%) } & \multicolumn{3}{c}{ Obesidad (\%) } \\
\hline $\begin{array}{l}\text { Edad } \\
\text { (años) }\end{array}$ & $\mathbf{N}$ & $\mathbf{N}(\%)$ & $\begin{array}{c}\text { Hombres } \\
\%\end{array}$ & $\begin{array}{c}\text { Mujeres } \\
\%\end{array}$ & $\mathbf{N}(\%)$ & $\begin{array}{c}\text { Hombres } \\
\%\end{array}$ & $\begin{array}{c}\text { Mujeres } \\
\%\end{array}$ \\
\hline 2 & 96 & $6(6,3)$ & 10,3 & 3,5 & $2(2,1)$ & 2,6 & 1,8 \\
\hline 3 & 89 & $9(10,1)$ & 16,2 & 5,8 & $4(4,5)$ & 10,8 & - \\
\hline 4 & 98 & $2(12,2)$ & 10,0 & 13,8 & $9(9,2)$ & 17,5 & 3,4 \\
\hline 6 & 136 & $17(12,5)$ & 13,2 & 12,0 & $19(14,0)$ & 20,8 & 9,6 \\
\hline 8 & 111 & $14(12,6)$ & 10,9 & 13,8 & $24(21,6)$ & 26,1 & 18,5 \\
11 & 145 & $23(15,9)$ & 11,9 & 18,6 & $23(15,9)$ & 20,3 & 12,8 \\
\hline 14 & 142 & $25(17,6)$ & 20,0 & 15,9 & $18(12,7)$ & 16,7 & 9,8 \\
\hline 18 & 153 & $28(18,3)$ & 26,56 & 12,35 & $11(7,2)$ & 6,25 & 7,9 \\
\hline
\end{tabular}

$N$ : tamaño muestral.

lencia de la suma de ambos a los ocho años (34,2\% de la muestra) (figura 1).

Respecto al riesgo de presentar obesidad a la edad de 18 a 19 años para un niño que era obeso en edades previas, este es evidente para obesidades detectadas desde los seis años, es máximo a los 11 años (figura 2) y mayor para ni- ñas que para niños, como se detalla en la tabla 3.

\section{Discusión}

Las cifras encontradas en nuestro estudio: prevalencia de obesidad del $7,2 \%$ de la muestra $(6,3 \%$ de los hombres y $7,9 \%$ de las mujeres) y sobrepeso del $18,3 \%$ 
Figura 1. Prevalencia de exceso de peso (sobrepeso más obesidad [\%]) para ambos sexos y a cada una de las edades consideradas.

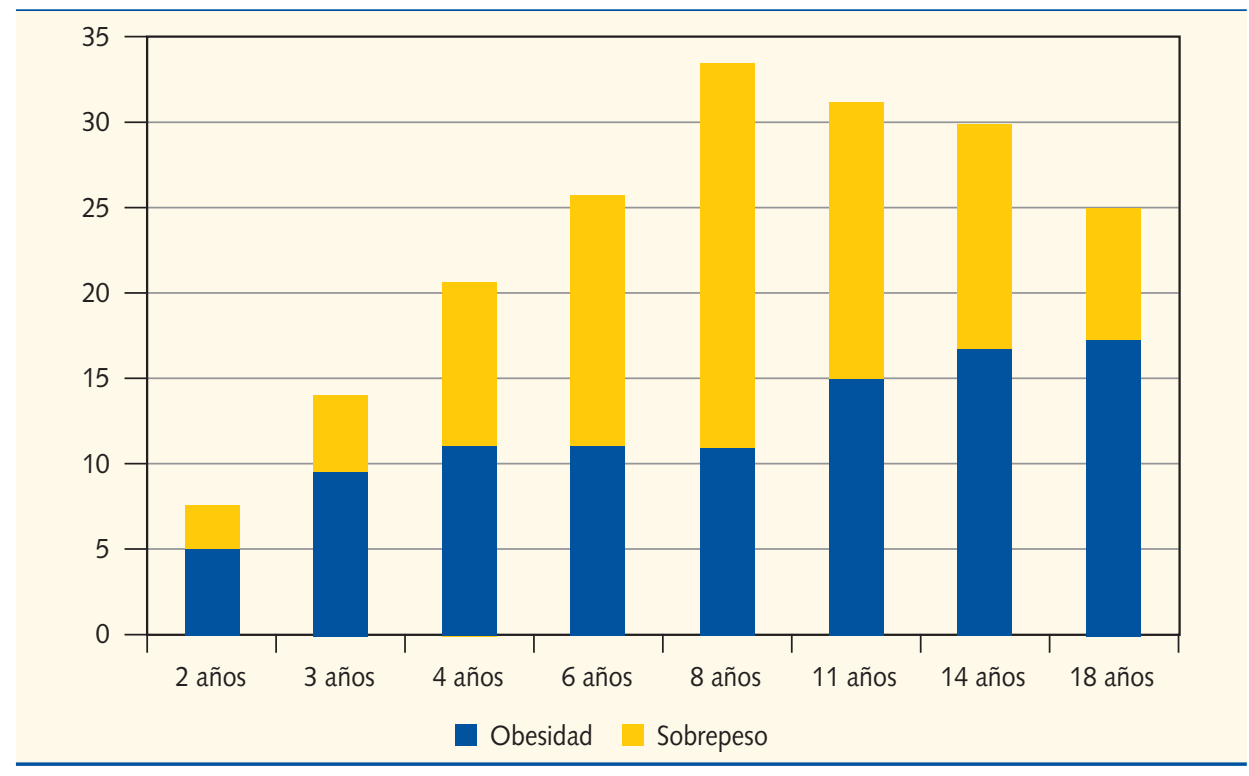

Figura 2. Riesgo de presentar obesidad a los 18 años en sujetos que presentan obesidad en edades previas según la edad en que se presenta, para la muestra total (\%).

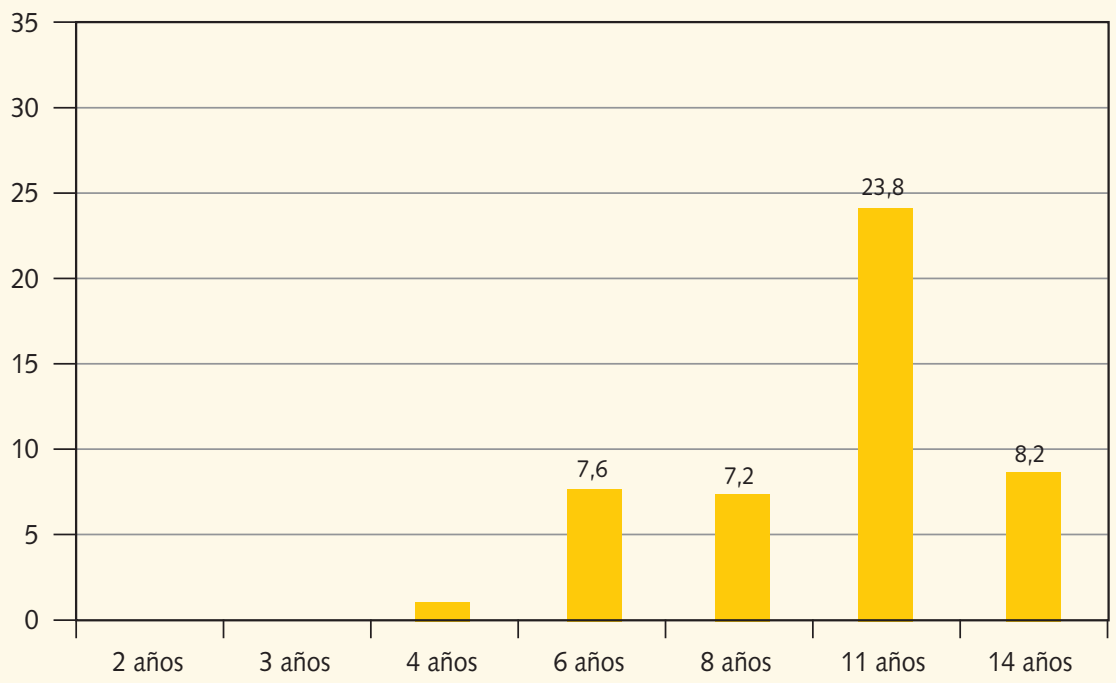


Tabla 3. Riesgo de presentar obesidad a la edad de 18 a 19 años para un niño obeso en edades previas

\begin{tabular}{l|c|c|c|c|c|c}
\hline & \multicolumn{2}{|c|}{ Total } & \multicolumn{2}{c|}{ Mujeres } & \multicolumn{2}{c}{ Hombres } \\
\hline Edad & RR & IC 95\% & RR & IC 95\% & RR & IC 95\% \\
\hline 4 & 1,236 & $0,174-8,798$ & 4,667 & $0,96-22,628$ & - & - \\
6 & 7,697 & $2,268-26,128$ & 12,500 & $3,381-46,212$ & 3,818 & $0,259-56,312$ \\
8 & 7,250 & $1,956-26,874$ & 8,833 & $1,824-42,778$ & 5,667 & $0,563-57,002$ \\
\hline 11 & 23,870 & $5,510-103,396$ & 40,909 & $5,426-308,452$ & 11,750 & $1,338-103,175$ \\
\hline 14 & 8,267 & $2,810-24,317$ & 12,333 & $3,337-45,581$ & 5,000 & $0,795-31,439$ \\
\hline
\end{tabular}

IC 95\%: intervalo de confianza del 95\%; RR: riesgo relativo.

(26,6\% de los hombres y $12,4 \%$ de las mujeres), consideradas conjuntamente, suponen que $25,5 \%$ de la muestra (uno de cada cuatro jóvenes) presenta un IMC superior al normal, más los hombres $(32,8 \%)$ que las mujeres $(20,22 \%)$. Son datos bastante parecidos a los publicados en el estudio EnKid para el grupo de edad de 18 a 24 años, en el que el $27,5 \%$ de los hombres y el $22,5 \%$ de las mujeres presentan sobrepeso u obesidad, aunque sus datos de obesidad son superiores a los nuestros e inferiores los de sobrepeso ${ }^{23}$. Las tablas de referencia utilizadas en el estudio EnKid son las de Hernández y cols., que tienen puntos de corte a los 18 años inferiores para obesidad y muy aproximados para sobrepeso, en hombres, e inferiores tanto en sobrepeso como en obesidad para mujeres. En otros estudios españoles, Larrañaga y cols., para el grupo de 15 a 18 años, refieren una prevalencia de sobrepeso del 18,2\%, para obesidad del $4,1 \%$ y conjunta del
$22,3 \%^{24}$; en el estudio AVENA, en adolescentes de 13 a 18,5 años, las cifras conjuntas de sobrepeso y obesidad son del $25,69 \%$ en hombres y del $19,13 \%$ en mujeres, y las de obesidad sola del $5,68 \%$ en hombres y del 3,08\% en mujeres ${ }^{25}$. En el estudio de Cuenca, en población ligeramente más joven, la prevalencia de sobrepeso es muy variable, del $15,7 \%$ en hombres y del $51,8 \%$ en mujeres, y la de obesidad es del 2,9\% en hombres y del $6,1 \%$ en mujeres ${ }^{26}$. En estos trabajos se utilizan las tablas internacionales, o de Cole, cuyos puntos de corte desde los 18 años son los mismos que los del presente trabajo. De especial interés consideramos los datos de la última Encuesta $\mathrm{Na}$ cional de Salud publicada, con resultados en población de entre 18 y 24 años muy similares a los obtenidos por nosotros: sobrepeso en el $18 \%$ (22,58\% de los hombres y $12,99 \%$ de las mujeres) y obesidad en el 5,5\% (5,53\% de los hombres y $5,46 \%$ de las mujeres $)^{27}$. Datos superpo- 
nibles a los de la Encuesta Europea de Salud 2009, también entre 18 y 24 años: $17,59 \%$ de sobrepeso $(22,05 \%$ en hombres y $12,90 \%$ en mujeres) y $5,73 \%$ de obesidad $(6,33 \%$ en hombres y $5,11 \%$ en mujeres $)^{28}$. En ambas encuenstas, las definiciones de obesidad y sobrepeso coinciden con la utilizada por nosotros. En el estudio DORICA, entre 25 y 34 años, la prevalencia de obesidad es del $5,9 \%$ (7,1\% en hombres, $4,8 \%$ en mujeres) y del $28,1 \%$ la de sobrepeso $(36,8 \%$ en hombres y $20,2 \%$ en mujeres ${ }^{29}$. Po- demos decir que nuestros resultados no difieren de los encontrados por otros autores españoles en muestras más grandes de población de edades comparables. Estos datos, reflejados en la tabla 4, corroboran que en España la prevalencia de obesidad y sobrepeso es alta.

Como reflejan estos estudios, salvo el de Cuenca, en estas edades la prevalencia de sobrepeso, pero no de obesidad, suele ser mayor en hombres que en mujeres, quizá por la mayor preocupación entre las chicas adolescentes

\begin{tabular}{|c|c|c|c|c|c|c|c|c|}
\hline Lugar & Ref. & Año & $\mathrm{N}$ & $\begin{array}{l}\text { Edad } \\
\text { (años) }\end{array}$ & Criterio & Sobrepeso \% & Obesidad \% & $\begin{array}{l}\text { Sobrepeso + } \\
\text { obesidad \% }\end{array}$ \\
\hline Este estudio & & 2008 & 153 & $18-19$ & Inter. Cole & $\begin{array}{l}18,3(26,6 \mathrm{H} ; \\
12,4 \mathrm{M})\end{array}$ & $\begin{array}{l}7,2(6,3 \mathrm{H} \\
7,9 \mathrm{M})\end{array}$ & $\begin{array}{l}25,5(32,8 \mathrm{H} ; \\
20,22 \mathrm{M})\end{array}$ \\
\hline $\begin{array}{l}\text { España } \\
\text { EnKID }\end{array}$ & 24 & $\begin{array}{l}1998- \\
2000\end{array}$ & 1477 & $18-24$ & $\begin{array}{l}\text { Obesidad: } \\
\text { IMC } \geq P_{97} \\
\text { Sobrepeso: } \\
I M C \geq P_{85} \\
y<P_{97}\end{array}$ & $\begin{array}{l}13,2(14,9 \mathrm{H} ; \\
11,3 \mathrm{M})\end{array}$ & $\begin{array}{l}13,7(12,6 \mathrm{H} ; \\
14,9 \mathrm{M})\end{array}$ & $\begin{array}{l}26,9\left(27,5 \mathrm{H}_{i}\right. \\
26,2 \mathrm{M})\end{array}$ \\
\hline País Vasco & 25 & $\begin{array}{l}2004- \\
2005\end{array}$ & 360 & $15-18$ & Inter. & 18,2 & 4,1 & 22,3 \\
\hline $\begin{array}{l}\text { España } \\
\text { AVENA }\end{array}$ & 26 & $\begin{array}{l}2000- \\
2002\end{array}$ & 2320 & $13-18$ & Inter. Cole & $\begin{array}{l}20,01 \mathrm{H} \\
16,05 \mathrm{M}\end{array}$ & $\begin{array}{l}5,68 \mathrm{H} ; \\
3,08 \mathrm{M}\end{array}$ & $\begin{array}{l}25,69 \mathrm{H} ; \\
19,13 \mathrm{M}\end{array}$ \\
\hline Cuenca & 27 & 1998 & - & $15-17$ & Inter. Cole & $\begin{array}{l}15,7 \mathrm{H} \\
51,8 \mathrm{M}\end{array}$ & $\begin{array}{l}2,9 \mathrm{H} \\
6,1 \mathrm{M}\end{array}$ & - \\
\hline $\begin{array}{l}\text { España } \\
\text { ENSE } 2006\end{array}$ & 28 & 2006 & - & $18-24$ & $\begin{array}{l}I M C \geq 25 y \\
<30 ; I M C \geq 30\end{array}$ & $\begin{array}{l}18(22,58 \mathrm{H} \\
12,99 \mathrm{M})\end{array}$ & $\begin{array}{l}5,5(5,53 \mathrm{H} ; \\
5,46 \mathrm{M})\end{array}$ & - \\
\hline $\begin{array}{l}\text { España } \\
\text { EES } 2009\end{array}$ & 29 & 2009 & - & $18-24$ & $\begin{array}{l}I M C \geq 25 y \\
<30 ; I M C \geq 30\end{array}$ & $\begin{array}{l}17,59(22,05 \\
H ; 12,90 M)\end{array}$ & $\begin{array}{l}5,73(6,33 \mathrm{H} ; \\
5,11 \mathrm{M})\end{array}$ & - \\
\hline $\begin{array}{l}\text { España } \\
\text { DORICA }\end{array}$ & 30 & - & - & $25-34$ & $\begin{array}{l}I M C \geq 25 \\
y<30 \\
I M C \geq 30\end{array}$ & $\begin{array}{l}28,1(36,8 \mathrm{H} ; \\
20,2 \mathrm{M})\end{array}$ & $\begin{array}{l}5,9(7,1 \mathrm{H} ; \\
4,8 \mathrm{M})\end{array}$ & - \\
\hline
\end{tabular}

EES: Encuesta Europea de Salud; ENSE: Encuesta Nacional de Sanidad; H: hombres; IMC: índice de masa corporal; Inter.: tablas internacionales; M: mujeres; N: tamaño muestral; Ref.: referencia bibliográfica. 
por su imagen, de hecho, en nuestro estudio un mayor porcentaje $(24,7 \%$ frente a $14,1 \%$ ) de ellas han sido objeto de alguna intervención dirigida al control de peso, y por la existencia de una mayor masa muscular en varones a estas edades.

En nuestra serie, la obesidad alcanza su más alta prevalencia a la edad de ocho años y disminuye en edades posteriores, mientras que el sobrepeso mantiene un ascenso continuado desde los dos hasta los cuatro años, permanece estable entre los seis y los $11 \mathrm{y}$ vuelve a elevarse a partir de esa edad. Considerando el exceso de peso en conjunto y para el total de la serie, la cifra más alta se observa a los ocho años, dato comparable al del estudio del País Vasco, con una mayor prevalencia entre los siete y los diez años ${ }^{24}, \mathrm{y}$ más precoz que el del estudio EnKid, entre los diez y los 13 años ${ }^{23}$, evolución parecida a la de niñas americanas con aumento de las cifras hasta los 12 años $^{18}$. En estas edades, la comparación entre distintos estudios está dificultada por la utilización en ellos de distintas tablas y/o distintos puntos de corte para definir obesidad y sobrepeso, también hay que considerar la edad de la población estudiada $y$, teniendo en cuenta la evolución al alza que se registra en el tiempo, la fecha en que se realizaron las medidas.

Es conocido el riesgo existente de perpetuar o mantener un estado de sobrepeso y/u obesidad presente en la infancia hasta la edad adulta con riesgos están en cifras variables según los autores $^{26,30}$ y mejorando la predicción a medida que se consideran edades infantiles más altas ${ }^{18,31}$. Sería muy interesante poder cuantificar el riesgo existente según en qué momento de la infancia se observe la obesidad, y determinar una edad en la cual, o a partir de la cual, los datos obtenidos ofrezcan una mayor fiabilidad.

Nuestros datos muestran aumento del riesgo a partir de los cuatro años en niñas y desde los seis años para ambos sexos, y no se observa a las edades más bajas (dos, tres y cuatro años para niños, y dos y tres años para niñas), quizá por el bajo número de casos con que contamos. Son más altas para niñas que para niños, siendo los 11 años la edad en que se registra un mayor riesgo, sobre todo en niñas, $y$, si bien coinciden en general con la tendencia recogida en la literatura en cuanto a que aumenta el riesgo a mayor edad, se cumple en nuestra serie hasta los 11 años, pero no a los 14 , edad para la que el riesgo es menor. Este dato podría estar en rela- 
ción con los cambios puberales registrados en esta edad en niñas, y no en niños, sobre todo en relación con la posición y distribución de la grasa y la ausencia a esta edad de una preocupación excesiva por la imagen corporal, que se iniciaría algo más tarde. En el estudio de Cuenca, los autores concluyen que el IMC en la infancia (9-12 años) tiene estrecha relación con el IMC en la adolescencia (14-17 años), siendo de 2,9 (IC 95\%: 2,21-4,04) el RR de que los niños con sobrepeso u obesidad en la primera exploración presentaran sobrepeso u obesidad seis años más tar$\mathrm{de}^{26}$. En el estudio Bogalusa, el riesgo de que el sobrepeso en adolescentes se mantenga en jóvenes adultos de etnia negra es del $62 \%$ en mujeres y del $52 \%$ en hombres ${ }^{30}$. Para Guo et al., la predicción es excelente con obesidades presentes a los 18 años, buena a los 13 y solo moderada para las obesidades existentes antes de los 13 años y cuantifica en 34 y $37 \%$ (para hombres y mujeres, respectivamente) la probabilidad de presentar sobrepeso a la edad de 35 años si a los 18 el IMC se situaba en el percentil $60^{31}$. Datos similares a este autor son los que refieren Thompson et al. en niñas y adolescentes hasta los 18 años, en las que es más probable presentar obesidad a los 21-23 años, si se ha presentado en edades previas, con una odds ratio (OR) de 11 a 30 según la edad en que se presente obesidad, siendo máxima a los 18 años y en ascenso desde los $13^{18}$. La probabilidad de ser adulto obeso (IMC mayor o igual a 30) es mayor o igual al $50 \%$ entre los chicos mayores de 13 años cuyo IMC es igual o excede el valor del percentil 95 para su edad y género ${ }^{32}$.

La principal limitación del estudio se debe al tamaño de la muestra, que dificulta la extrapolación de los datos obtenidos a muestras más amplias de población y quizá dificulte también una exhaustiva interpretación de algunos resultados. Se debe al origen de la misma: casos procedentes de las consultas de Pediatría de un centro de salud seguidos durante 16-17 años. Este prolongado seguimiento es causa de un elevado número de pérdidas. Se trata además de una edad en que la sensación de salud disminuye e incluso provoca rechazo al sistema sanitario, cabe preguntarse si alguno de los que rechazaron participar lo haría por no querer afrontar la existencia de un problema relacionado con el peso, y en ese caso la muestra presentaría un sesgo. De todos modos, es muy interesante comprobar cómo a pesar de esta limitación los datos obtenidos guardan relación con los obtenidos recientemen- 
te en muestras españolas más amplias y de características similares. El origen mayoritariamente español de los participantes se debe a que en el inicio del seguimiento la inmigración era un fenómeno considerablemente menor que en la actualidad, y hace que los datos no sean aplicables a la generalidad de nuestra población actual, sino solo a la de origen español, dada la distinta prevalencia de obesidad y sobrepeso en personas de distintas etnias.

Podemos concluir que la obesidad y el sobrepeso son patologías frecuentes en jóvenes adultos en nuestro medio, re- gistrándose un riesgo importante de presentar obesidad en este momento en niños obesos que lo eran desde los seis años de edad.

Creemos que este trabajo aborda un problema de salud de especial relevancia en la actualidad, particularmente en la Atención Primaria, y especialmente en Pediatría. Tiene un alto interés para los profesionales del centro y para la población que atiende por ser este el ámbito adecuado para prevenir, detectar precozmente y tratar esta patología y aquellas para las que es factor de riesgo.

\section{Bibliografía}

1. Kopelman P. Health risks associated with overweight and obesity. Obesity Rev. 2007;8(Sup 1):13-1.

2. Dietz W. Chidhood Weight Affects Adult Morbidity and Mortality. J Nutr. 1998;128:411S-4S.

3. Van Dam RM, Willett WC, Manson JE, Hu FB, Van Dam RM, Willett WC et al. The relationship between overweight in adolescence and premature death in women. Ann Intern Med. 2006; 18(145):91-7.

4. Leis $\mathrm{R}$, Martínez AA, Novo AA, Villar HH, Zimmo $S$. Cardiovascular risk factors among obese children and adolescents. The GALINUT Study. J Pediatr Gastroenterol Nutr. 2004;39 (Suppl 1):468-9.

5. Csabi G, Torok K, Jeges S, Molnar D. Presence of metabolic cardiovascular syndrome in obese children. Eur J Pediatr. 2000;159:91-4.
6. Cook S, Weitzman $M$, Auinger $P$, Nguyen $M$, Dietz WH. Prevalence of a metabolic syndrome phenotipe in adolescents: Findings from the Third National Health and Nutrition Examination Survey 19881994. Arch Pediatr Adolesc Med. 2003;157: 821-7.

7. Viner R, Segal T, Lichtarowicz-Krynska E, Hindmarsh P. Prevalence of the insulin resistanse sindrome in obesity. Arch Dis Child. 2005;90:10-4.

8. Kimm SY, Barton BA, Obarzanek E, McMahon RP, Sabry ZI, Waclawiw MA et al. Racial divergence in adiposity during adolescence: The NHLBI Growth and Healt Study. Pediatrics. 2001; 107:e34.

9. Lobstein T, Baur L, Uauy R. Obesity in children and young people. IASO. Obesity Rev. 2004; 5(Suppl 1):S4-85.

10. Onis $M$, Blossner $M$. Prevalence and trends of overweight among preschool children in developing countries. Am J Clin Nutr. 2000;72:1032-9. 
11. Duelo Marcos M, Escribano Ceruelo E, Muñoz Velasco F. Obesidad. Rev Pediatr Aten Primaria. 2009;11(Supl 16):s239-57.

12. Wang $Y$, Lobstein T. Worldwide trends in childhood overweight and obesity. Int J Pediatr Obes. 2006;1:11-25.

13. Benson L, Baer HJ, Kaelber DC. Trends in the diagnosis of Overweight and Obesity in Children and Adolescents: 1999-2007. Pediatrics. 2009;123:e153-8.

14. Rubio MA, Salas-Salvadó J, Barbany M, Moreno $B$, Aranceta J, Bellido D y cols. Consenso SEEDO 2007 para la evaluación del sobrepeso y la obesidad y el establecimiento de criterios de intervención terapéutica. Rev Esp Obes. 2007;5(3): 135-75.

15. Mei Z, Grumer-Strawn LM, Pietrobelli $A$, Goulding A, Goran M, Dietz W. Validity of body mass index compared with other body-composition screening indexes for the assessment of body fatness in children and adolescencents. Am J Clin Nutr. 2002;75:978-85.

16. Grupo de trabajo de la guía sobre la prevención y el tratamiento de la obesidad infantojuvenil. Centro Cochrane Iberoamericano, coordinador. Guía de práctica clínica sobre la prevención y el tratamiento de la obesidad infantojuvenil. Madrid: Plan de Calidad para el Sistema Nacional de Salud del Ministerio de Sanidad y Política Social. Agència d'Avaluació de Tecnologia i Recerca Mèdiques; 2009. Guía de práctica clínica: AATRM N. ${ }^{\circ}$ 2007/ 25.

17. Sun Guo S, Wu W, Cameron W, Roche A. Predicting overweight and obesity in adulthood from body mass index values in childhood and adolescence. Am J Clin Nutr. 2002;76:653-8.

18. Thompson D, Obarzanek E, Franco D, Barton B, Morrison J, Biro F et al. Childhood Overweight and Cardiovascular Disease Risk Factors: The National Heart, Lung, and Blood Institute
Growth and health Study. J Pediatr. 2007;150:18 25.

19. Freedman DS, Khan LK, Serdula MK, Dietz WH, Srinivasan SR, Berenson GS. The relation of childhood BMI to adult adiposity: the Bogalusa heart Study. Pediatrics. 2005;115:22-7.

20. Wisemandle W, Maynard M, Guo SS, Siervogell RM. Childhood weight, stature, and body mass index among never overweight, early-onset overweight, and late-onset overweight groups. Pediatrics. 2000;106:e14.

21. Albañil Ballesteros $M R$, Sánchez Martín $M$, de la Torre Verdú $M$, Olivas Domínguez A, Sánchez Méndez MY, Sanz Cuesta MT. Prevalencia de obesidad a los 14 años en cuatro consultas de atención primaria. Evolución desde los dos años. An Pediatri (Barc). 2005;63(1):39-44.

22. Albañil Ballesteros MR, Sánchez Martín $M$, Sanz Cuesta T. Prevalencia de obesidad en una consulta de Pediatría de Atención Primaria. Evolución del índice de masa corporal. Rev Pediatr Aten Primaria. 2007:9:219-30.

23. Serra Majem LI, Ribas Barba L, Aranceta Bartrina J, Pérez Rodrigo C, Saavedra Santana P, Peña Quintana L. Obesidad infantil y juvenil en España. Resultados del Estudio enKid (1998-2000). Med Clin (Barc). 2003;121:725-32.

24. Larrañaga $N$, Amiano $P$, Arrizabalaga JJ, Bidaurrazaga J, Gorostiza E. Prevalence of obesity in 4-18-year-old population in the Basque Country, Spain. Obesity Rev. 2007;8:281-7.

25. Moreno LA, Mesana MI, Fleta J, Ruiz JR, González-Gros $M$, Sarría $A$ et al. The AVENA Study Group. Overweight, Obesity and Body Fat Composition in Spanish Adolescents. Ann Nutr Metab. 2005;49:71-6.

26. Martínez Vizcaíno F, Salcedo Aguilar F, Rodríguez Artalejo F, Martínez Vizcaíno V, Domínguez Contreras LM, Torrijos Regidor R. Prevalencia de la obesidad y mantenimiento del estado ponde- 
ral tras un seguimiento de 6 años en niños y adolescentes: estudio de Cuenca. Med Clin (Barc). 2002;119:327-30.

27. Encuesta Nacional de Salud de España 2006. Ministerio de Sanidad y Consumo, Instituto Nacional de Estadística. Disponible en www.msc.es/ estadEstudios/estadisticas/encuestaNacional/ encuestaNac2006/EstilosVidaPorcentaje.pdf

28. Instituto Nacional de Estadística. Encuesta Europea de Salud 2009. Índice de masa corporal según sexo y grupo de edad. Población de 18 y más años. Disponible en www.ine.es/jaxi/tabla.do

29. Aranceta Bartrina J, Serra Majem LL, Foz-Sala $M$, Moreno Esteban B y grupo colaborativo SEEDO. Prevalencia de obesidad en España. Med Clin (Barc). 2005;125:460-6.
30. Srinivasan SR, Bao W, Wattigney WA, Berenson GS. Adolescent overweight is associated with adult overweight and related multiple cardiovascular risk factors: the Bogalusa Heart Study. Metabolism. 1996;45:235-40.

31. Guo S, Chumlea WC. Tracking of body mass index in children in relation to overweight in adulthood. Am J Clin Nutr. 1999;70(Suppl):145S$8 \mathrm{~S}$.

32. Whitlock EP, Williams SB, Gold R, Smith PR, Shipman SA. Screening and interventions for childhood overweight: a summary of evidence for the US Preventive Services Task Force. Pediatrics. 2005;116:e125-44. 